\title{
Parametric vis-a-vis non parametric measures describing $G$ x $E$ interactions for salt salinity tolerant barley genotypes in multi-environment trials
}

\author{
Ajay Verma* \\ Statistics and Computer Center, ICAR-Indian Institute of Wheat and Barley \\ Research, Karnal-132001 (Haryana), India \\ V. Kumar \\ ICAR-Indian Institute of Wheat and Barley Research, Karnal-132001 (Haryana), India \\ A.S. Kharab \\ ICAR-Indian Institute of Wheat and Barley Research, Karnal-132001 (Haryana), India \\ R Chatrath \\ ICAR-Indian Institute of Wheat and Barley Research, Karnal-132001 (Haryana), India \\ G.P. Singh \\ ICAR-Indian Institute of Wheat and Barley Research, Karnal-132001 (Haryana), India \\ ${ }^{*}$ Corresponding author. E-mail: verma.dwr@gmail.com
}

\begin{abstract}
GXE interaction to know adaptability of 19 salt salinity tolerant barley genotypes was studied by parametric and non-parametric measures. Genotypes KB1516, RD2907 and RD2794 showed minimum environmental variance over different environments. Superiority index identified genotypes RD2907 and NDB1445 with lowest value accompanied with higher. Wricke's measure exhibited lower values of DWRB168, DWRB165 and NDB1445. Higher values of GAl showed consistent performance of RD2907, NDB1445 and RD2552. Non-parametric measures $S_{i}{ }^{(1)}, S_{i}{ }^{(3)}$ and $S_{i}{ }^{(6)}$ the considered DWRB165 and DWRB168 as desirable genotypes. Thennarasu's first measure $\mathrm{NP}_{\mathrm{i}}{ }^{(1)}$ found DWRB168 and NDB1445 as desirable adaptable and KB1546, RD2907 and NDB1173 were unstable genotypes. Wricke's parameter was positively correlated with $N P_{i}^{(1)}, N P_{i}^{(3)}$ and Kang. GAl had significant positive with $P_{i}$ and Kang while negative with $\mathrm{S}_{\mathrm{i}(6)}, N \mathrm{NP}_{\mathrm{i}(2)}$ \& $N \mathrm{P}_{\mathrm{i}(4)}$. Worth to mention the negative association of $\mathrm{P}_{\mathrm{i}}$ with $\mathrm{S}_{\mathrm{i}(6)}, N \mathrm{NP}_{\mathrm{i}}^{(2),} N \mathrm{NP}_{\mathrm{i}}$ (4). Non parametric measures $\mathrm{S}_{\mathrm{i}}{ }^{(3)} \mathrm{S}_{\mathrm{i}}{ }^{(6)} \mathrm{NP}_{\mathrm{i}}{ }^{(2)}$ \& $\mathrm{NP}_{\mathrm{i}}{ }^{(4)}$ clubbed together while Kang, $\mathrm{W}_{\mathrm{i}}{ }^{2}$, $\mathrm{S}^{2}{ }_{i} \mathrm{~S}_{\mathrm{i}}{ }^{(1)}, \mathrm{S}_{\mathrm{i}}{ }^{(2)} N \mathrm{NP}_{\mathrm{i}}{ }^{(1)} \& N \mathrm{NP}_{\mathrm{i}}{ }^{(3)}$ joined in another cluster. Left over parametric measures were grouped in two separate clusters i.e. $\left(b_{i}, S^{2}{ }_{x i}, C V_{i}\right),(Y i e l d, G A I P i)$ respectively. Biplot analysis based on first two principal components showed three groups among the measures.
\end{abstract}

Keywords: Adaptability G x E interaction, Parametric and nonparametric measures, Salinity tolerant barley

\section{INTRODUCTION}

Genotype x environment interactions had been exploited for better adaptation of genotypes in a broad range of environments (Baxevanos et al., 2008). Genotypes with stable trait expression across environments contribute little to GxE interaction and performance would be predictable from the main effects of genotypes and environments (Henryk et al., 2014). Statistical methods have been proposed for the adaptability analysis, with the prime aim to explain the GxE interaction (Dehghani et al., 2016). Mostly two approaches

\section{Article Info}

DOI:10.31018/jans.v10i2.1736

Received: December 29, 2017

Revised: January 25, 2018

Accepted: March 28, 2018

\section{How to Cite}

Verma, A. et al. (2018).

Parametric vis-a-vis non parametric measures describing $G \times E$ interactions for salt salinity tolerant barley genotypes in multienvironment trials. Journal of Applied and Natural Science, 10(2): 557-563 
phenomenon of genotype by environment interaction (Van Eeuwijk et al., 2001). Now a days breeding programs are incorporating elements of both parametric and nonparametric measures (Mohammadi and Amri, 2008, Sisay and Sharma, 2016).

Prime objectives of the study were to (1) analyze GxE interactions on yield of 19 barley genotypes under salt salinity trials (2) identify barley genotypes that have high yield and stable performance across different environments (3) study the relationship among parametric and non parametric measures.

\section{MATERIALS AND METHODS}

Parametric measures i.e. Regression coefficient (Finlay and Wilkinson, 1963), Environmental variance (Becker and Leon, 1988), Shukla variance (1972), Ecovalence (Wricke's, 1962), Coefficient of variation (Francis and Kanenberg 1978), Superiority index (Lin and Binns, 1988), Geometric adaptability index (Mohammadi and Amri, 2008), Kang's rank sum (1988) ; were studied to estimate $\mathrm{g} x$ e interaction for nineteen salt salinity tolerant barley genotypes evaluated at 06 locations. Treatments were laid in field trials by Randomized block design with four replications. Recommended agronomical practices were utilized to harvest the good crop and yield was considered for further analysis. Non parametric measures of Hühn and Nassar (1989) were considered to study adaptability behavior proposed based on ranks of genotypes and use the idea of homeostasis as measure of the stability. Additionally four non parametric measures of Thennarasu's (1995) based on adjusted ranks of genotypes within each test environments. Spearman's rank correlation analysis (Piepho \& Lotito, 1992) estimates the correlation among ranks as follows :

$$
\bar{r}_{s}=1-\frac{6 \sum_{t=1}^{n} d_{i}^{2}}{n\left(n^{2}-1\right)}
$$

where $d_{i}$ denotes difference between ranks for $i$-th genotype and $\mathrm{n}$ is total number of pairs.

Stable genotype would be with regression coefficient $b_{i}$ equals to one. GxE interaction effect for $i-$ th genotype, squared and summed across environments to obtain Wricke's Ecovalence measure. Low ecovalence value indicates high relative stability, greatest stability is when $\mathrm{W}_{i}^{2}=0$. Environmental variance is a measure for static concept of stability and a genotype with minimum $S_{x 2}^{i}$ under different environments is considered to be stable. The stability was also measured through combining mean yield and coefficient of variation, genotypes with low $\mathrm{CV}_{i}$ and high mean yield were considered as most desirable. Superiority measure $P_{i}$ is the mean square of distance between $i$ thgenotype and the genotype with maximum yield within each environment. A low value of $P_{i}$ indicates high relative stability. Geometric mean can be use as a measure of adaptability of genotype which called as geometric adaptability index (GAI). Genotypes will high GAl will be desirable. Spearman's rank correlation was calculated to measure the relationship among the statistical measures using SAS software and principal component analysis (PCA) were performed by JMP (2007) Software to comprehend the relationships among the statistics. For hierarchical clustering the Euclidean distance was used as a dissimilarity measure required in Ward's (Ward, 1963) clustering method. SAS-based computer program SASGESTAB (Hussein et al., 2000) employed to calcu-late nonparametric measures.

\section{RESULTS AND DISCUSSION}

Main effects of environment $(E)$, genotype $(G)$ and $G \times E$ interaction were highly significant $P<0.01$ as per analysis of variance. The mean yield of genotypes over environments was ranged from 40.9 to 30.7 along with grand mean yield of $35.76 \mathrm{q} / \mathrm{ha}$. Ten genotypes out of nineteen with yield more than grand mean yield. Since the GXE interaction was significant, the average yield of the genotypes was subjected to further adaptability analysis (Truberg and Hühn, 2000). According to Finlay and Wilkinson (1963) all the genotypes had $b_{i}$ near 1.0 , indicating average stability over environments. As per environmental variance $\left(S^{2}{ }_{x i}\right)$ the KB1516, RD2907 and RD2794 with minimum variance over different environments were considered to be stable while RD2958 and RD2956 considered being unstable genotypes (Sisay and Sharma, 2016).

By using Francis and Kannenberg's (1978) stability parameter $\left(C_{i}\right)$ the genotypes KB1546, RD2907 and RD2794 considered to be stable with different average yields other hand, RD2958, NDB1173 and RD2794 with high CVi considered to be unstable genotypes. Superiority index $\left(P_{\mathrm{i}}\right)$ identified genotypes RD2907 and NDB1445 with the highest yield considered to be stable while RD2958 and KB1546 with the highest $P_{i}$ value were the unstable genotypes along with the lower yield (Tables 3 and 4) (Dehghani et al., 2016). According to Wricke's (1962) stability parameter $\left(W_{i}^{2}\right)$ the genotypes DWRB168, DWRB165 and NDB1445 with lower ecovalance were considered to be stable and RDB2958, KB1546, and RD2552 with high ecovalance were unstable genotypes. On the basis of GAI RD2907, NDB1445 and RD2552 ranked as three stable genotypes and RD2958 and DWRB165 as unstable genotypes (Mohammadi and Amri, 2008).

Significance of $S_{i}{ }^{(1)}$ and $S_{i}^{(2)}$ were tested as per Hühn and Nassar (1989). For each genotype, $Z_{1}$ and $Z_{2}$ values were calculated based on the ranks of adjusted data and then summed: $Z_{1}$ sum = 24.17 and $Z_{2}$ sum $=17.30$ (Table 5 ). Both these statistics are distributed as $c^{2}$ and were less than the critical value of $x^{2}(0.01,19)=30.6$. This indi- 
Verma A. et al. / J. Appl. \& Nat. Sci. 10 (2): 557 - 563 (2018)

Table 1. Parentage details and environmental conditions.

\begin{tabular}{|c|c|c|c|c|c|c|c|}
\hline Code & Genotype & Parentage & Code & Environments & Latitude & Longitude & Altitude (m) \\
\hline$\overline{G 1}$ & KB1507 & RD 2742/K 877 & E 1 & Hisar & $29^{\circ} 10^{\prime} \mathrm{N}$ & $75^{\circ} 46^{\prime} \mathrm{E}$ & 215.2 \\
\hline G 2 & KB1523 & K 508/ RD 2676 & E 2 & Faizabad-I & $26^{\circ} 47^{\prime} \mathrm{N}$ & $82^{\circ} 12^{\prime} \mathrm{E}$ & 113 \\
\hline G 3 & KB1546 & IBYT-HI-08 (2013-14) & E 3 & Faizabad-II & $26^{\circ} 47^{\prime} \mathrm{N}$ & $82^{\circ} 12^{\prime} \mathrm{E}$ & 113 \\
\hline G 4 & NDB1655 & EIBGN-66 (2008-09) & E 4 & Dalipnagar & $26.59^{\prime} \mathrm{N}$ & $79.18^{\prime} \mathrm{E}$ & 145 \\
\hline G 5 & NDB1665 & 1st GSBSN-32 (2013-14) & E 5 & Banasthali & $26.40^{\prime} \mathrm{N}$ & $75.87^{\prime} \mathrm{E}$ & 287.27 \\
\hline G 6 & NDB1673 & 1st GSBSN-106 (2013-14) & E 6 & DWR Hisar & $29^{\circ} 10^{\prime} \mathrm{N}$ & $75^{\circ} 48^{\prime} \mathrm{E}$ & 215 \\
\hline G 7 & HUB258 & EMBSN-27/RD2503 & & & & & \\
\hline G 8 & DWRB165 & PETUNIA.1/LAMOLIN95 & & & & & \\
\hline G 9 & DWRB168 & $\begin{array}{l}\text { EXCEL-BAR/4/GLORIA-BAR/ } \\
\text { COME//LIGNEE640/3/SPB }\end{array}$ & & & & & \\
\hline G 10 & BH1017 & NBGSN-13 (2009)/DWRB73 & & & & & \\
\hline G 11 & RD2907 & RD103/RD2518//RD2592 & & & & & \\
\hline G 12 & RD2955 & RD2666/DWR46 & & & & & \\
\hline G 13 & RD2956 & DL472/BL2//RD2508 & & & & & \\
\hline G 14 & RD2957 & RD2552/RD2786 & & & & & \\
\hline G 15 & RD2958 & RD2552/RD2786 & & & & & \\
\hline G 16 & RD2552 & RD2035/DL472 & & & & & \\
\hline G 17 & NDB1173 & BYTLRA 3-(1994-95)/NDB217 & & & & & \\
\hline G 18 & NDB1445 & NDB940/Ratna & & & & & \\
\hline G 19 & RD2794 & RD2035/RD2683 & & & & & \\
\hline
\end{tabular}

Table 2. Parametric measures of GxE interactions.

\begin{tabular}{|c|c|c|c|c|c|c|c|c|}
\hline Genotype & Yield & $b_{i}$ & $S^{2} x i$ & $\mathbf{C V}_{\mathrm{i}}$ & $\mathbf{W}_{\mathrm{i}}{ }^{2}$ & $\mathbf{s}_{i}^{2}$ & GAl & $\mathbf{P}_{\mathrm{i}}$ \\
\hline KB1507 & 38.47 & 0.9980 & 142.90 & 31.07 & 134.12 & 28.71 & 37.03 & 32.38 \\
\hline KB1523 & 34.59 & 0.9983 & 127.94 & 32.70 & 43.20 & 8.39 & 33.10 & 64.60 \\
\hline KB1546 & 31.92 & 0.9864 & 24.30 & 15.45 & 267.01 & 58.41 & 31.60 & 114.58 \\
\hline NDB1655 & 37.20 & 1.00 & 217.00 & 39.60 & 87.72 & 18.34 & 34.84 & 47.21 \\
\hline NDB1665 & 37.72 & 1.00 & 231.04 & 40.29 & 64.00 & 13.04 & 35.29 & 37.56 \\
\hline NDB1673 & 36.92 & 1.00 & 219.44 & 40.12 & 87.32 & 18.25 & 34.25 & 46.81 \\
\hline HUB258 & 34.63 & 1.00 & 205.93 & 41.43 & 80.62 & 16.75 & 31.96 & 67.69 \\
\hline DWRB165 & 30.71 & 0.9959 & 98.30 & 32.28 & 40.20 & 7.72 & 29.53 & 113.67 \\
\hline DWRB168 & 34.06 & 1.000 & 152.64 & 36.27 & 20.74 & 3.37 & 32.16 & 70.43 \\
\hline $\mathrm{BH} 1017$ & 31.89 & 0.9950 & 105.94 & 32.27 & 139.81 & 29.98 & 30.29 & 106.74 \\
\hline RD2907 & 40.92 & 0.9914 & 59.50 & 18.85 & 131.59 & 28.14 & 40.33 & 19.68 \\
\hline RD2955 & 34.61 & 1.00 & 229.01 & 43.72 & 120.40 & 25.64 & 31.67 & 74.93 \\
\hline RD2956 & 37.11 & 1.00 & 236.30 & 41.42 & 131.48 & 28.12 & 35.04 & 45.40 \\
\hline RD2957 & 36.58 & 0.9978 & 127.62 & 30.88 & 68.24 & 13.98 & 35.41 & 44.28 \\
\hline RD2958 & 31.28 & 1.00 & 269.39 & 52.47 & 371.25 & 81.72 & 28.25 & 125.01 \\
\hline RD2552 & 39.81 & 0.9999 & 169.13 & 32.66 & 144.34 & 30.99 & 38.09 & 26.03 \\
\hline NDB1173 & 32.97 & 1.00 & 233.90 & 46.39 & 131.00 & 28.01 & 30.06 & 97.67 \\
\hline NDB1445 & 40.85 & 1.00 & 167.09 & 31.64 & 40.83 & 7.86 & 39.08 & 20.53 \\
\hline RD2794 & 37.16 & 0.9956 & 97.74 & 26.60 & 60.45 & 12.24 & 36.16 & 44.41 \\
\hline
\end{tabular}

Table 3. Non - parametric measures of GxE interactions.

\begin{tabular}{|c|c|c|c|c|c|c|c|c|c|c|c|c|}
\hline & Yield & $S_{i}{ }^{(1)}$ & $Z_{1}$ & $S_{i}^{(2)}$ & $Z_{2}$ & $\mathbf{S}_{i}^{(3)}$ & $S_{i}{ }^{(6)}$ & $\mathrm{NP}_{\mathrm{i}}{ }^{(1)}$ & $\mathrm{NP}_{\mathrm{i}}{ }^{(2)}$ & $\mathrm{NP}_{\mathrm{i}}{ }^{(3)}$ & $\mathrm{NP}_{\mathrm{i}}{ }^{(4)}$ & Kang \\
\hline$\overline{\text { KB1507 }}$ & 7.33 & 4.27 & 1.75 & 13.07 & 2.18 & 8.91 & 2.18 & 4.67 & 0.5833 & 5.72 & 1.05 & 19 \\
\hline KB1523 & 12.00 & 5.73 & 0.14 & 26.00 & 0.12 & 10.83 & 2.17 & 3.67 & 0.2529 & 5.19 & 0.52 & 17 \\
\hline KB1546 & 12.50 & 7.67 & 0.76 & 40.30 & 0.81 & 16.12 & 2.64 & 5.83 & 0.4321 & 6.34 & 0.60 & 34 \\
\hline NDB1655 & 8.50 & 7.40 & 0.49 & 37.10 & 0.38 & 21.82 & 3.65 & 5.17 & 0.6078 & 5.77 & 0.89 & 16 \\
\hline NDB1665 & 7.17 & 4.73 & 1.05 & 14.97 & 1.72 & 10.44 & 2.42 & 4.17 & 0.6410 & 4.82 & 0.84 & 11 \\
\hline NDB1673 & 8.67 & 7.07 & 0.24 & 34.67 & 0.17 & 20.00 & 3.23 & 4.67 & 0.6667 & 5.83 & 0.83 & 18 \\
\hline HUB258 & 11.83 & 6.33 & 0.00 & 27.37 & 0.05 & 11.56 & 1.97 & 5.00 & 0.4167 & 5.62 & 0.61 & 19 \\
\hline DWRB165 & 15.83 & 3.27 & 3.88 & 9.37 & 3.24 & 2.96 & 0.84 & 3.33 & 0.1961 & 3.65 & 0.34 & 21 \\
\hline DWRB168 & 12.33 & 3.87 & 2.50 & 10.27 & 2.96 & 4.16 & 1.30 & 2.50 & 0.2174 & 2.87 & 0.31 & 15 \\
\hline $\mathrm{BH} 1017$ & 13.33 & 7.07 & 0.24 & 34.67 & 0.17 & 13.00 & 2.10 & 5.50 & 0.3667 & 6.18 & 0.64 & 33 \\
\hline RD2907 & 5.83 & 5.53 & 0.26 & 23.37 & 0.33 & 20.03 & 3.94 & 5.83 & 1.2963 & 6.21 & 1.28 & 15 \\
\hline RD2955 & 11.33 & 6.80 & 0.10 & 30.27 & 0.00 & 13.35 & 2.29 & 5.50 & 0.4783 & 6.15 & 0.71 & 23 \\
\hline RD2956 & 8.67 & 6.27 & 0.00 & 27.07 & 0.07 & 15.62 & 3.00 & 4.50 & 0.5000 & 5.55 & 0.92 & 21 \\
\hline RD2957 & 9.33 & 5.60 & 0.21 & 21.87 & 0.50 & 11.71 & 2.57 & 5.33 & 0.5079 & 5.56 & 0.70 & 17 \\
\hline RD2958 & 12.17 & 7.13 & 0.28 & 34.17 & 0.13 & 14.04 & 2.22 & 4.67 & 0.3457 & 5.99 & 0.60 & 37 \\
\hline RD2552 & 5.67 & 6.27 & 0.00 & 31.47 & 0.02 & 27.76 & 4.47 & 4.50 & 1.2857 & 5.25 & 1.07 & 20 \\
\hline NDB1173 & 13.00 & 5.07 & 0.65 & 19.60 & 0.82 & 7.54 & 1.69 & 5.83 & 0.5303 & 7.15 & 0.66 & 27 \\
\hline NDB1445 & 3.83 & 3.53 & 3.23 & 8.97 & 3.37 & 11.70 & 3.39 & 2.83 & 0.8095 & 3.94 & 1.04 & 5 \\
\hline RD2794 & 8.67 & 10.80 & 8.39 & 24.27 & 0.25 & 14.00 & 2.62 & 4.17 & 0.4386 & 5.43 & 1.64 & 12 \\
\hline & & Sum $=$ & 24.17 & & 170 & & & & $x^{2}(0.05,1)$ & 3.84 & $x^{2}(0.01,1)$ & 6.63 \\
\hline$E\left(s^{1}\right)$ & 6.3158 & $E\left(s^{2}\right)$ & 30.0 & $\mathrm{~V}\left(\mathrm{~s}^{1}\right)$ & 2.3956 & $V\left(s^{2}\right)$ & 131.40 & & $x^{2}(0.05,19)$ & 30.1 & $x^{2}(0.01,19)$ & 36.2 \\
\hline
\end{tabular}


Verma A. et al. / J. Appl. \& Nat. Sci. 10 (2): 557 - 563 (2018)

Table 4. Ranking of genotypes by parametric vis-à-vis non parametric measures.

\begin{tabular}{|c|c|c|c|c|c|c|c|c|c|c|c|c|c|c|c|c|c|c|}
\hline & Yield & $\mathbf{b}_{\mathrm{i}}$ & $S^{2} x i$ & $\mathbf{C V}_{\mathrm{i}}$ & $\mathbf{W}_{\mathrm{i}}{ }^{2}$ & $\mathrm{~s}^{2} \mathrm{i}$ & GAI & $\mathbf{P i}$ & $S_{i}{ }^{(1)}$ & $\mathrm{S}_{\mathrm{i}}{ }^{(2)}$ & $\mathrm{S}_{\mathrm{i}}{ }^{(3)}$ & $S_{i}{ }^{(6)}$ & $\mathrm{NP}_{\mathrm{i}}{ }^{(1)}$ & $\mathrm{NP}_{\mathbf{i}}{ }^{(2)}$ & $\mathbf{N P}_{\mathbf{i}}{ }^{(3)}$ & $\mathbf{N P}_{\mathbf{i}}{ }^{(4)}$ & Kang & SRT \\
\hline KB1507 & 4 & 12 & 8 & 5 & 15 & 15 & 4 & 4 & 4 & 4 & 4 & 7 & 11 & 13 & 11 & 16 & 11 & 148 \\
\hline KB1523 & 13 & 5 & 7 & 10 & 4 & 4 & 11 & 11 & 9 & 10 & 6 & 6 & 4 & 3 & 5 & 3 & 8 & 119 \\
\hline KB1546 & 16 & 1 & 1 & 1 & 18 & 18 & 15 & 18 & 18 & 19 & 15 & 13 & 19 & 7 & 18 & 5 & 18 & 220 \\
\hline NDB1655 & 6 & 16 & 13 & 12 & 10 & 10 & 9 & 10 & 17 & 18 & 18 & 17 & 13 & 14 & 12 & 13 & 6 & 214 \\
\hline NDB1665 & 5 & 17 & 16 & 14 & 6 & 6 & 7 & 5 & 5 & 5 & 5 & 10 & 6 & 15 & 4 & 12 & 2 & 140 \\
\hline NDB1673 & 9 & 13 & 14 & 13 & 9 & 9 & 10 & 9 & 15 & 17 & 16 & 15 & 11 & 16 & 13 & 11 & 9 & 209 \\
\hline HUB258 & 11 & 10 & 12 & 16 & 8 & 8 & 13 & 12 & 12 & 12 & 7 & 4 & 12 & 6 & 10 & 6 & 11 & 170 \\
\hline DWRB165 & 19 & 4 & 4 & 8 & 2 & 2 & 18 & 17 & 1 & 2 & 1 & 1 & 3 & 1 & 2 & 2 & 14 & 101 \\
\hline DWRB168 & 14 & 7 & 9 & 11 & 1 & 1 & 12 & 13 & 3 & 3 & 2 & 2 & 1 & 2 & 1 & 1 & 5 & 88 \\
\hline $\mathrm{BH} 1017$ & 17 & 2 & 5 & 7 & 16 & 16 & 16 & 16 & 15 & 16 & 10 & 5 & 16 & 5 & 16 & 7 & 17 & 202 \\
\hline RD2907 & 1 & 3 & 2 & 2 & 14 & 14 & 1 & 1 & 7 & 8 & 17 & 18 & 19 & 19 & 17 & 18 & 5 & 166 \\
\hline RD2955 & 12 & 14 & 15 & 17 & 11 & 11 & 14 & 14 & 13 & 13 & 11 & 9 & 16 & 9 & 15 & 10 & 15 & 219 \\
\hline RD2956 & 8 & 19 & 18 & 15 & 13 & 13 & 8 & 8 & 11 & 11 & 14 & 14 & 8 & 10 & 8 & 14 & 14 & 206 \\
\hline RD2957 & 10 & 11 & 6 & 4 & 7 & 7 & 6 & 6 & 8 & 7 & 9 & 11 & 14 & 11 & 9 & 9 & 8 & 143 \\
\hline RD2958 & 18 & 18 & 19 & 19 & 19 & 19 & 19 & 19 & 16 & 15 & 13 & 8 & 11 & 4 & 14 & 4 & 19 & 254 \\
\hline RD2552 & 3 & 9 & 11 & 9 & 17 & 17 & 3 & 3 & 11 & 14 & 19 & 19 & 8 & 18 & 6 & 17 & 12 & 196 \\
\hline NDB1173 & 15 & 15 & 17 & 18 & 12 & 12 & 17 & 15 & 6 & 6 & 3 & 3 & 19 & 12 & 19 & 8 & 16 & 213 \\
\hline NDB1445 & 2 & 8 & 10 & 6 & 3 & 3 & 2 & 2 & 2 & 1 & 8 & 16 & 2 & 17 & 3 & 15 & 1 & 101 \\
\hline RD2794 & 7 & 6 & 3 & 3 & 5 & 5 & 5 & 7 & 19 & 9 & 12 & 12 & 6 & 8 & 7 & 19 & 3 & 136 \\
\hline
\end{tabular}

Table 5. Loading of parametric and non parametric measures.

\begin{tabular}{|c|c|c|}
\hline Measure & PCA 1 & PCA 2 \\
\hline $\begin{array}{l}\text { Yield } \\
\end{array}$ & 0.3827 & -0.1156 \\
\hline $\mathrm{Bi}$ & 0.0081 & 0.0757 \\
\hline$S^{2}{ }_{x i}$ & 0.0744 & 0.0519 \\
\hline $\mathrm{CV}_{\mathrm{i}}$ & 0.1842 & -0.0153 \\
\hline Wi 2 & 0.1237 & 0.3621 \\
\hline$s^{2}{ }_{i}$ & 0.1237 & 0.3621 \\
\hline GAI & 0.3968 & -0.0686 \\
\hline $\mathrm{Pi}$ & 0.3960 & -0.0464 \\
\hline$S_{i}{ }^{(1)}$ & 0.1293 & 0.2869 \\
\hline$S_{i}{ }^{(2)}$ & 0.1642 & 0.3199 \\
\hline$S_{i}^{(3)}$ & -0.0808 & 0.3646 \\
\hline$S_{i}^{(6)}$ & -0.2589 & 0.2837 \\
\hline$N P_{i}{ }^{(1)}$ & 0.1324 & 0.3090 \\
\hline $\mathrm{NP}_{\mathrm{i}}{ }^{(2)}$ & -0.2976 & 0.2216 \\
\hline$N P_{i}^{(3)}$ & 0.1696 & 0.3180 \\
\hline $\mathrm{NP}_{\mathrm{i}}{ }^{(4)}$ & -0.3175 & 0.2095 \\
\hline Kang & 0.3410 & 0.1452 \\
\hline$\%$ variance & 34.89 & 32.25 \\
\hline
\end{tabular}

cated the non-significant differences among genotypes as per ranks of $S_{i}^{(1)}$ and $S_{i}^{(2)}$ measures (Elahe and Ebadi, 2015). More over the individual $Z$ values showed RD2794 \& DWRB165 were significantly unstable relative to others, with $Z_{i}$ (1) val- ues more than the critical value of $x^{2}(0.05,1)=$ 3.84 .

Results of non-parametric stability statistics showed that considering to $S_{i}^{(1)}, S_{i}{ }^{(3)}$ and $S_{i}^{(6)}$ the genotypes DWRB165 and DWRB168 were the stable genotypes but had the low mean yield. Based on $S_{i}^{(3)}, S_{i}^{(6)}$ the genotypes NDB1173 apart from DWRB168 were of stable performance but had the lower yield (Tables 3 and 4). All of these non-parametric statistics were identified NDB1665 and RD2552 as unstable genotypes. According to Thennarasu's (1995) nonparametric measures, which considered ranks of adjusted yield, genotypes with minimum low values are considered more stable. Based on the first measure $\mathrm{NP}_{i}^{(1)}$ DWRB168 and NDB1445 were stable and KB1546, RD2907 and NDB1173 were unstable genotypes. According to the other three methods $\left(\mathrm{NP}_{\mathrm{i}}{ }^{(2)}, \quad \mathrm{NP}_{\mathrm{i}}{ }^{(3)}\right.$ and $\left.\mathrm{NP}_{\mathrm{i}}{ }^{(4)}\right)$ genotypes DWRB168 and DWRB165 were stable and the genotypes RD2907 and RD2552 were unstable (Baxevanos et al., 2008). Most of cases these measures selected genotypes with low average yield as stable genotypes.

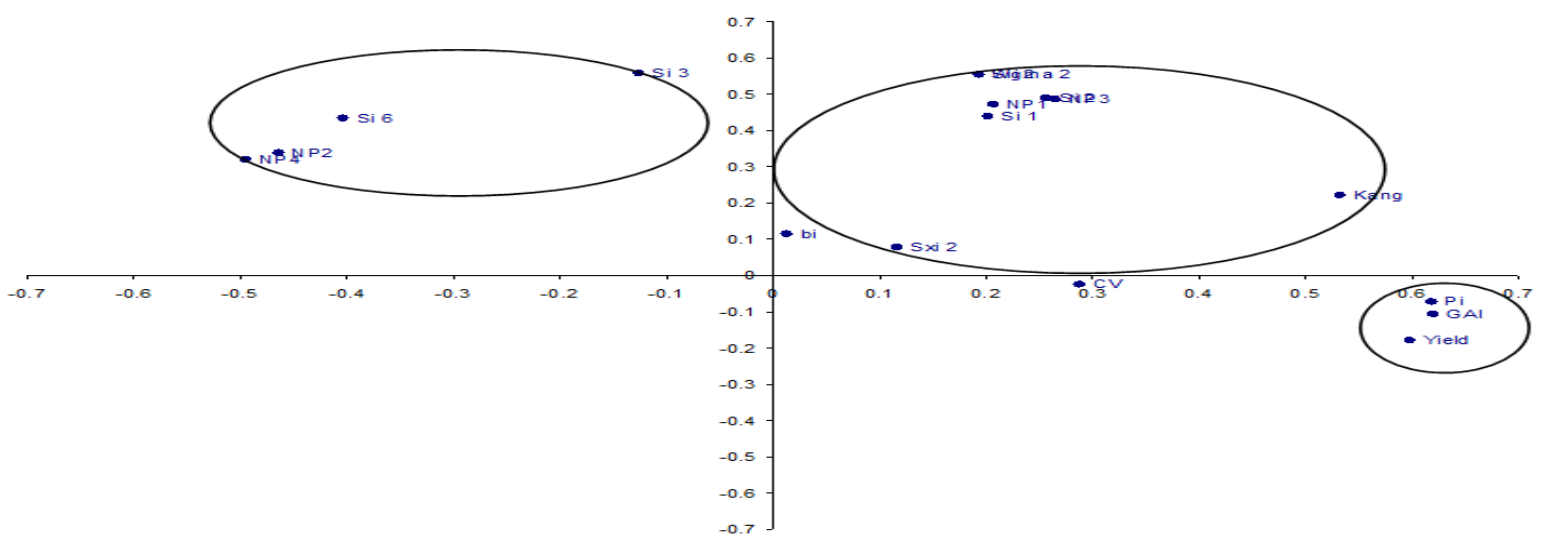

Fig. 1. Biplot analysis of parametric and non parametric measures of $G \times E$. 
Table 6. Association analysis among measures.

\begin{tabular}{|c|c|c|c|c|c|c|c|c|c|c|c|c|c|c|c|c|}
\hline$b_{i}$ & $\begin{array}{l}\text { Yield } \\
-0.2158\end{array}$ & bi & Sxi 2 & $\mathrm{CV}_{\mathrm{i}}$ & $\mathbf{W}_{\mathrm{i}}{ }^{2}$ & $\mathbf{s}^{2}{ }_{i}$ & GAI & $\mathbf{P i}$ & $S_{i}{ }^{(1)}$ & $\mathbf{S}_{\mathrm{i}}{ }^{(2)}$ & $\mathbf{S}_{i}^{(3)}$ & $S_{i}^{(6)}$ & $\mathbf{N P}_{\mathbf{i}}{ }^{(1)}$ & $\mathbf{N P}_{\mathbf{i}}{ }^{(2)}$ & $\mathbf{N P}_{\mathbf{i}}{ }^{(3)}$ & $\mathbf{N P}_{\mathbf{i}}{ }^{(4)}$ \\
\hline $\mathrm{S}^{2} \mathrm{xi}$ & -0.2298 & 0.9930 & & & & & & & & & & & & & & \\
\hline $\mathrm{CV}_{\mathrm{i}}$ & 0.0877 & 0.9193 & 0.9140 & & & & & & & & & & & & & \\
\hline$W_{i^{2}}^{2}$ & 0.1333 & 0.1246 & 0.1596 & 0.1368 & & & & & & & & & & & & \\
\hline $\mathrm{s}^{2}{ }_{i}$ & 0.1333 & 0.1246 & 0.1596 & 0.1368 & 1.0000 & & & & & & & & & & & \\
\hline GAI & 0.9509 & 0.0281 & 0.0140 & 0.3351 & 0.2281 & 0.2281 & & & & & & & & & & \\
\hline $\mathrm{Pi}$ & 0.9719 & -0.2053 & -0.2316 & 0.0789 & 0.1561 & 0.1561 & 0.9421 & & & & & & & & & \\
\hline$S_{i}^{(1)}$ & 0.2246 & 0.0351 & -0.0193 & -0.0298 & 0.3316 & 0.3316 & 0.1947 & 0.2947 & & & & & & & & \\
\hline $\mathrm{S}_{\mathrm{i}}^{(2)}$ & 0.2474 & 0.0561 & -0.0035 & 0.0193 & 0.4474 & 0.4474 & 0.2772 & 0.3474 & 0.8930 & & & & & & & \\
\hline $\mathrm{S}_{\mathrm{i}}{ }^{(3)}$ & -0.3351 & 0.0667 & 0.0070 & -0.1491 & 0.3140 & 0.3140 & -0.3070 & -0.2193 & 0.6912 & 0.7456 & & & & & & \\
\hline $\mathrm{S}_{\mathrm{i}}{ }^{(6)}$ & -0.7053 & 0.1035 & 0.0614 & -0.2228 & 0.0667 & 0.0667 & -0.6842 & -0.5912 & 0.3246 & 0.3544 & 0.8491 & & & & & \\
\hline$N P_{i}{ }^{(1)}$ & 0.1105 & 0.1263 & 0.1298 & 0.1684 & 0.6368 & 0.6368 & 0.2053 & 0.1719 & 0.3175 & 0.4158 & 0.2140 & -0.0263 & & & & \\
\hline $\mathrm{NP}_{\mathrm{i}}{ }^{(2)}$ & -0.8105 & 0.2877 & 0.2807 & 0.0000 & 0.0526 & 0.0526 & -0.7298 & -0.7158 & -0.0421 & 0.0456 & 0.5281 & 0.7877 & 0.1386 & & & \\
\hline$N P_{i}{ }^{(3)}$ & 0.1158 & 0.2070 & 0.2333 & 0.1982 & 0.6825 & 0.6825 & 0.1965 & 0.1456 & 0.3982 & 0.4421 & 0.2456 & 0.0263 & 0.8070 & 0.1895 & & \\
\hline $\mathrm{NP}_{\mathrm{i}}{ }^{(4)}$ & -0.8158 & 0.1649 & 0.1754 & -0.1456 & 0.0579 & 0.0579 & -0.8193 & -0.7754 & 0.0982 & -0.0281 & 0.4965 & 0.7368 & 0.0526 & 0.8421 & 0.2035 & \\
\hline Kang & 0.7149 & 0.0535 & 0.0728 & 0.2816 & 0.7465 & 0.7465 & 0.7798 & 0.6991 & 0.2377 & 0.3851 & -0.0711 & -0.4202 & 0.5430 & -0.4009 & 0.5588 & -0.4781 \\
\hline
\end{tabular}

Critical values of correlation at $5 \%$ and $1 \%$ level of significance are 0.4853 and 0.6152 respectively.

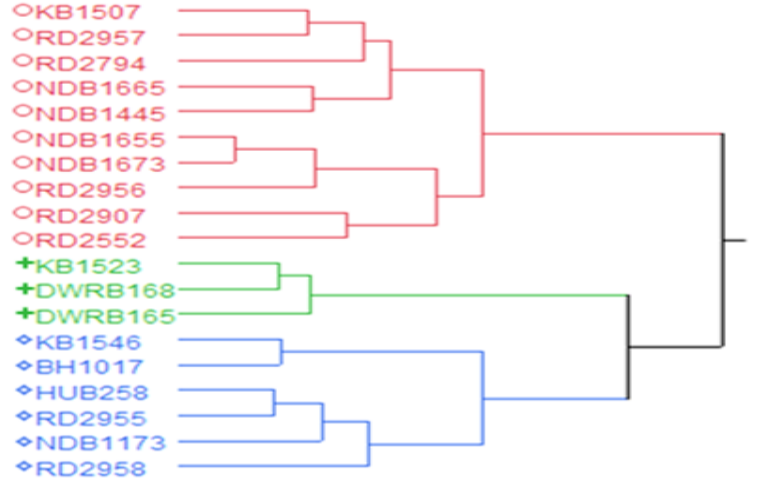

Fig. 2. Dendogram of salt salinity tolerant barley genotypes.

Interrelationship among parametric and nonparametric measures: Spearman's rank correlation (Table 5) among measures exhibited high positive correlation of yield with two parametric measures $\left(P_{i}, G A I\right)$ and Kang measure that is expected as the low values of $P_{i}$ and high values of GAl were related to high yielder genotypes (Sisay and Sharma, 2016). Negative significant correlation of yield with most of nonparametric measures except $\mathrm{NP}_{i}^{(1)} \mathrm{NP}_{\mathrm{i}}^{(3)}$ suggested that selection of stable genotypes based on these statistics should be considered seriously with genotype yield. The regression coefficient $\left(b_{i}\right)$ was positively and strongly correlated with $S^{2}{ }_{x i}$ and $C V_{i}$. Environmental variance $\left(S^{2}{ }_{x i}\right)$ was significantly and positively correlated with $\mathrm{CV}_{\text {i. }}$. Wricke's parameter $\left(\mathrm{W}_{\mathrm{i}}{ }^{2}\right)$ was positively correlated with $\mathrm{NP}_{\mathrm{i}}{ }^{(1)}, \mathrm{NP} \mathrm{P}_{\mathrm{i}}$ ${ }^{(3)}$ and Kang. $\mathrm{S}^{2}$ maintained high linear relation with $\mathrm{NP}_{\mathrm{i}}{ }^{(1)}, \mathrm{NP}_{\mathrm{i}}{ }^{(3)}$ and Kang and moderate with $\mathrm{S}_{\mathrm{i}}$ (1),$S_{i}^{(2)}$ GAl had significant positive with $P_{i}$ and Kang while negative with $\mathrm{S}_{i}^{(6)}, \mathrm{NP}_{i}{ }^{(2)} \& \mathrm{NP}_{i}{ }^{(4)}$. Worth to mention the negative association of $\mathrm{P}_{i}$ with $\mathrm{S}_{i}^{(6),} N P_{i}^{(2),} N P_{i}^{(4)}$

Similar results reported by Mohammadi and Amri (2008). Nassar and Hühn (1987) reported that $S^{2}{ }_{x i}$, $S_{i}^{(1)}$ and $S_{i}^{(2)}$ are associated with the static or biological concept of stability. Flores et al. (1998) categorized $S^{2 \times i}, S_{i}^{(1)}$ and $S_{i}^{(2)}$ in same group and de-

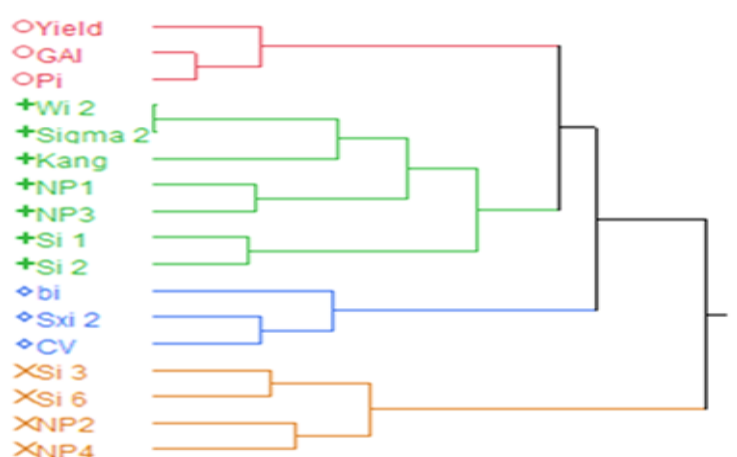

Fig. 3. Clustering of parametric and non parametric measures.

fined them in the sense of homeostasis. Piepho and Lotito (1992) reported high rank correlation among parametric and non-parametric measures. Truberg and Huehn (2000) suggested an alternative use of non-parametric measures, such as stability variance whenever assumptions, such as normal distribution, independence, homogeneity of error variances, absence of outliers, etc. are violated.

Non-parametric measures $\mathrm{S}_{i}^{(\mathrm{s})}$ were positively and significantly correlated among themselves and with Thennarasu's $\mathrm{NP}_{i}^{(\mathrm{s})}$ measures also mentioned by Hühn and Nassar (1989). NP ${ }_{i}^{\text {(s) }}$ also showed strong positive correlation among themselves. The positive correlation of Kang with parametric and nonparametric measures except $\mathrm{S}_{i}^{(1)} \mathrm{NP}_{\mathrm{i}}^{(2)} \mathrm{NP}_{\mathrm{i}}^{(4)}$ indicated similar aspects of stability by these measures. Therefore, it is possible to Kang only as one of the measure of adaptability.

Hierarchical clustering of genotypes and measures: Clustering of barley genotypes as per ranks of yields and GxE measures was performed. Output of analysis in form of dendrogram separated the genotypes into three clusters (Figure 2). The cluster of desirable genotypes DWRB165, DWRB 168, KB1523 identified by non parametric measures. Separate cluster of RD2958, RD2955, NDB 1173, KB 1546, BH1017, HUB258 were pointed out by para- 
metric measures. Large cluster of higher and moderate yielders genotypes mentioned by parametric and non parametric measures.

Attempt was made to find pattern if any among the measures of GxE interaction for considered salt salinity tolerant barley genotypes. Clustering mentioned four groups of studied measures. Non parametric measures $S_{i}{ }^{(3)} S_{i}{ }^{(6)} N P_{i}{ }^{(2)} \& N P_{i}{ }^{(4)}$ clubbed together while Kang, $\mathrm{Wi}^{2}, \mathrm{~S}^{2}{ }_{\mathrm{i}} \mathrm{S}_{\mathrm{i}}{ }^{(1)}, \mathrm{S}_{\mathrm{i}}$ (2) $\mathrm{NP}_{\mathrm{i}}{ }^{(1)} \& \mathrm{NP}_{\mathrm{i}}{ }^{(3)}$ joined in another cluster. Remaining parametric measures were grouped in two separate clusters i.e. $\left(b_{i}, S^{2}{ }_{x i}, C V_{i}\right),($ Yield, $\mathrm{GAI} \mathrm{Pi}$ ). This showed the clear difference of parametric measures from non parametric measures.

Biplot analysis of parametric and non parametric measures: Graphical display of the relationships among measures is displayed in a biplot of first two principal components (PC1 and PC2) as these PC's accounts for more than $67 \%$ of total variation. Major three groups to be distinguished as below:

Group I: GAI, $P_{i}$, Yield

Group II: $\mathrm{S}_{\mathrm{i}(3)}, \mathrm{S}_{\mathrm{i}(6)}, \mathrm{NP}_{\mathrm{i}(2)}, \mathrm{NP}_{\mathrm{i}(4)}$

GroupllI: $S^{2}{ }_{x i}, K a n g, N P_{i(1)}, N P_{i(3)}, S_{i(1)}, S_{i(2)}, W_{i}{ }^{2} s^{2}{ }_{i}$ Yield is included in group I, suggesting group I comprised those methods where yield had an important influence on the ranking across environments. According to this group genotypes RD2907, NDB1445, RD2552, and KB1507 introduced as stable genotypes that were the first five high yielding genotypes (Tables 3 and 4). There were strong positive rank correlation between these two measures and yield. Therefore, yield would be good measure for selection (Table 5). Superiority measure $\left(P_{i}\right)$ and GAl as measures of genotypic performance attempt to integrate both yield and stability. Selection based on these stability parameters is related to the dynamic or agronomic concept of stability.

Non-parametric measures $\mathrm{S}_{i}^{(3)}, \mathrm{S}_{i}^{\left({ }^{(6)}\right.}, \mathrm{NP}_{i}^{(2)}$ and $\mathrm{NP}_{\mathrm{i}}{ }^{(4)}$ were included in group II. These measures selected DWRB165 \& DWRB168 as stable genotypes that were the low yielding genotypes. The measures of this group were negatively correlated with mean yield. High yielder genotypes would be unstable as per these non parametric measures. This need further study in other crops also (Table 5).

Measures of Group III $\mathrm{S}_{\mathrm{xi}}^{2}, \mathrm{Kang}, \mathrm{NP}_{\mathrm{i}}^{(1)}, \mathrm{NP}_{\mathrm{i}}^{\left({ }^{(3)}\right.}, \mathrm{S}_{\mathrm{i}}$ (1) $, S_{i}{ }^{(2)}, W_{i}{ }^{2}, S_{i}^{2}$ identified NDB1655, BH1017, RD2958 were as undesirable genotypes for yield and specific adaptable behavior.

\section{Conclusion}

Parametric and nonparametric measures have been studied to quantify GxE interaction of 19 barley genotypes. Both yield and stable performance considered simultaneously to exploit the useful effect of GxE interaction in order to select promising genotypes. For salt affected area of the country, the availability of salinity tolerant genotypes with high yield is very much needed to insure good farmer income.

\section{ACKNOWLEDGEMENTS}

Authors sincerely acknowledge the efforts of the staff at testing centers within the Barley Network project to carry out the field evaluation of salt salinity tolerant barley genotypes and data recording.

\section{REFERENCES}

Baxevanos D, Goulas C, Tzortzios S, Mavromatis A (2008). Interrelationship among and repeatability of seven stability indices estimated from commercial cotton (Gossypium hirsutum L.) variety evaluation trials in three Mediterranean countries. Euphytica 161: 371-382

Becker HC, Leon J (1988). Stability analysis in plant breeding. Plant Breed 101:1-23

Dehghani MR, Majidi MM, Mirlohi A, Saeidi G. (2016). Integrating parametric and non-parametric measures to investigate genotype $\times$ environment interactions in tall fescue. Euphytica. 208:583-596.

Elahe Noruzi, Asghar Ebadi .(2015). Comparison of Parametric and Non-parametric Methods for Analysing Genotypex Environment Interactions in Sunflower (Helianthus annuus L.) Inbred Lines Jordan Journal of Agricultural Sciences, 11(4):959-978.

Finlay KW, Wilkinson GN (1963) The analysis of adaptation in a plant breeding programme. Aust $J$ Agric Res 14:742-754

Francis, T.R., Kannenberg L.W .1978. Yield stability studied in short-season maize. I. A descriptive method for grouping genotypes. Can J Plant Sci 58:10291034

Flores, F., M. T. Moreno, J. I. Cubero (1998). A comparison of univariate and multivariate methods to analyze G x E interaction. Field Crops Research, 56: 271286.

Henryk Bujak, Kamila Nowosad, Roman Warzecha (2014). Evaluation of maize hybrids stability using parametric and non-parametric methods Maydica 59: 170-175.

Hühn, M., Nassar, R. (1989). On tests of significance for nonparametric measures of phenotypic stability. Biometrics, 45: 997-1000.

Hussein, M.A., Bjornstad, A. and Aastveit, A.H. (2000). SASG $x$ ESTAB: A SAS program for computing genotype $x$ environment stability statistics. Agron. J., 92: 454-459.

Kang's, M.S. (1988). A rank-sum method for selecting high-yielding, stable corn genotypes. Cereal Res. Commun., 16:113-115.

Lin, C. S. and Binns M. R. (1988). A method of analyzing cultivar $\times$ location $\times$ year experiments: A new stability parameter. Theor. Appl. Genet., 76: 425430.

Mohammadi, R. and Amri A. (2008). A. Comparison of parametric and non-parametric methods for selecting stable and adapted durum wheat genotypes in variable environments. Euphytica, 159: 419-432.

Piepho, H.P., Lotito, S. (1992). Rank correlation among parametric and nonparametric measures of pheno- 
typic stability. Euphytica 64: 221-225.

Shukla, G.K. (1972). Some statistical aspects of partitioning genotype environmental components of variability. Heredity, 29:237-245.

Sisay Awoke and M.K. Sharma (2016). Parametric and Non-Parametric Methods to Describe Genotype by Environment Interaction and Grain Yield Stability of Bread Wheat Statistics and Applications. 14(1\&2): 9-29

Thennarasu's, K. (1995). On certain non-parametric procedures for studying genotype-environment interactions and yield stability. Unpublished Ph.D. Thesis. P.G. School, IARI, New Delhi

Truberg B. and Hühn, M. (2000). Contributions to the analysis of genotype $x$ environments interactions:
Comparison of different parametric and nonparametric tests for interactions with emphasis on crossover interaction. J. Agron. Crop Sci., 185:267274

Van Eeuwijk F.A., Cooper M., DeLacy I.H. (2001). Some vocabulary and grammar for the analysis of multienvironment trials, as applied to the analysis of FPB and PPB trials. Euphytica, 122:477-490

Ward, J.H. (1963). Hierarchical grouping to optimize an objective function. J Am Stat Assoc. 58:236-244.

Wricke's, G. (1962). On a method of understanding the biological diversity in field research. Z. Pflanzenzucht, 47: 92-96. 